\title{
Fully Automatic Teeth Segmentation in Adult OPG Images ${ }^{\dagger}$
}

\author{
Nicolás Vila-Blanco ${ }^{1, *}$, Inmaculada Tomás ${ }^{2}$ and María José Carreira ${ }^{1}$ \\ 1 Centro de Investigación en Tecnoloxías da Información (CITIUS), Universidade de Santiago de \\ Compostela, 15782 Santiago de Compostela, Spain; mariajose.carreira@usc.es \\ 2 Oral Sciences Research Group, Universidade de Santiago de Compostela, Health Research Institute \\ Foundation of Santiago (FIDIS), 15872 Santiago de Compostela, Spain; inmaculada.tomas@usc.es \\ * Correspondence: nicolas.vila@usc.es; Tel.: +34-8818-16445 \\ + Presented at the XoveTIC Congress, A Coruña, Spain, 27-28 September 2018.
}

Published: 17 September 2018

\begin{abstract}
In this work, the problem of segmenting teeth in panoramic dental images is addressed. The Random Forest Regression Voting Constrained Local Models (RFRV-CLM) are used to perform the segmentation in two steps. Firstly, a set of mandible and teeth keypoints are located, and then that points are used to initialise each individual tooth model. A method to detect missing teeth based on the quality of fit is presented. The system is evaluated using 346 manually annotated images containing adult-stage teeth. Encouraging results on detecting missing teeth are achieved. The system is able to locate the outline of the teeth to a median point-to-curve error of $0.2 \mathrm{~mm}$.
\end{abstract}

Keywords: teeth segmentation; panoramic dental images; random forest regression-voting; machine learning

\section{Introduction}

Since they discovery, dental X-ray images have been widely used in a variety of clinical fields, such as abnormality detection, treatment and surgery planning, prostheses design, assessment of children's dental development, human identification and many more. Extraoral panoramic images in particular show a full coverage of the teeth as well as other surrounding bones, such as the mandible or the vertebrae. However, the quality of these images is quite challenging to automatic processing algorithms, mainly because the acquisition process is highly dependent on the patient positioning and patient movements.

\section{Methods}

Our main contribution is the development of a fully automatic procedure to detect and outline mandibular adult-stage teeth in panoramic dental images, and a simple method to detect missing teeth. To do that, Random Forest Regression Voting Constrained Local Models (RFRV-CLM) are used. This method combines a global linear shape model with local appearance models to locate each shape point. Full details of the method are explained in [1].

One possible approach is to build an individual model for each specific tooth. Due to the symmetry of the mouth, the teeth models of one mandible side can be used to outline the teeth on the other side. However, there are two main limitations. First of all, the search space is too big when compared with the target teeth shapes, so the teeth models needs a reasonably good initialisation. Furthermore, the teeth are very close to each other and the shapes are very similar within each tooth type (incisors, molars, etc.), so the tooth search can easily converge to a neighbouring tooth. 
To overcome this problem, a two-step segmentation procedure is proposed. In the first step, a RFRV-CLM model is trained to detect a set of mandible and keypoints. This allows to capture the pose variation of teeth in relation with other teeth and with the mandible. Furthermore, the model initialization is easier due to the target shape occupies the great part of the image. In the second step, the initial shapes for each tooth model are calculated from the previous detected keypoints, and refined with the individual teeth models. Besides, a simple method based on the thresholding of the quality-of-fit per tooth is applied after the teeth shape search in order to detect missing teeth.

The full procedure was evaluated in a set of 346 panoramic images (261 images for training and 85 for testing). In each image, the shapes of seven left-mandibular teeth were manually annotated. The individual tooth models and the keypoint model were built with the RFRV-CLM algorithm. The predicted shapes of left-mandibular teeth were compared to ground truth and the performance was assessed in two ways.

Firstly, the missing teeth detection was evaluated as a classification problem with two target classes: missing (negative class) or present (positive class). The accuracy of the system was over $95 \%$, where the precision, sensitivity and specificity were $99 \%, 96 \%$ and $84 \%$, respectively.

Secondly, the accuracy of the teeth shape outlining was assessed with the point-to-curve error, which represents the shortest distance of each predicted shape point to the curve through the ground truth points. This measurement was obtained in correctly located teeth, i.e., the teeth whose predicted shapes overlaps with the ground truth shape more than $50 \%$. The results show a median error of less than $0.23 \mathrm{~mm}$ for all types of teeth and the $99 \%$ ile is $1.31 \mathrm{~mm}$ in the worst case, which demonstrate the robustness of this procedure.

Author Contributions: N.V.B. designed and developed the experiments, analysed the results and wrote the paper. I.T.C. provided the OPG database and validated the results from a clinical point of view. M.J.C.N. helped to design the experiments and analyse the results, and validated the results from a technical point of view.

Acknowledgments: This work has received financial support from the Consellería de Cultura, Educación e Ordenación Universitaria (accreditation 2016-2019, ED431G/08; ED431B 2017/029) and the European Regional Development Fund (ERDF).

Conflicts of Interest: The authors declare no conflict of interest. The founding sponsors had no role in the design of the study; in the collection, analyses, or interpretation of data; in the writing of the manuscript, and in the decision to publish the results.

\section{Reference}

1. Lindner, C.; Bromiley, P.A.; Ionita, M.C.; Cootes, T.F. Robust and accurate shape model matching using random forest regression-voting. IEEE Trans. Pattern Anal. 2015, 37, 1862-1874.

(C) 2018 by the authors. Licensee MDPI, Basel, Switzerland. This article is an open access article distributed under the terms and conditions of the Creative Commons Attribution (CC BY) license (http://creativecommons.org/licenses/by/4.0/). 